\title{
Intravenous fenoldopam for acute kidney injury
}

\author{
Yi Liu ${ }^{1}$ Fu Shan $\mathrm{Xue}^{2} \cdot$ Rui Ping $\mathrm{Li}^{2}$
}

Received: 14 July 2015 / Accepted: 20 July 2015 / Published online: 5 August 2015

(C) Japanese Society of Anesthesiologists 2015

Keywords Fenoldopam · Acute kidney injury · Orthotopic liver transplantation

\section{To the Editor:}

In a recent study by Biancofiore et al. [1] evaluating the effect of intravenous fenoldopam on acute renal injury (AKI) after orthotopic liver transplantation, AKI was determined according to the Acute Kidney Injury Network criteria within 7 days from surgery. In fact, the Acute Kidney Injury Network criteria require the use of a 48-h time window. Furthermore, it was unclear why only stage 2 AKI was included, though stages 1 and 3 AKI are also common after liver transplantation [2].

This single-arm prospective study showed that serum creatinine levels significantly decreased 48 and $72 \mathrm{~h}$ after intravenous fenoldopam. It has been shown that serum creatinine levels rapidly recover back to normal limits within 2 days or less in about $50 \%$ of patients with postoperative AKI [2]. The patients given fenoldopam in the current study showed better renal function parameters compared to a historical cohort including comparable AKI patients not given fenoldopam, but the authors did not specify whether

This letter refers to the article doi:10.1007/s00540-014-1951-2.

Fu Shan Xue

xuefushan@aliyun.com; fushan.xue@gmail.com

1 Department of Anesthesiology, Shanxi Province Tumor Hospital, Taiyuan and Wujiaqu People's Hospital, Wujiaqu City, Xinjiang, People's Republic of China

2 Department of Anesthesiology, Plastic Surgery Hospital, Chinese Academy of Medical Sciences and Peking Union Medical College, 33 Ba-Da-Chu Road, Shi-Jing-Shan District, Beijing 100144, People's Republic of China demographic and surgical variables were comparable between the two series. Furthermore, the small sample size of this study may not exclude a high risk of $\alpha$ statistical error. Because of these limitations, it is difficult to differentiate whether the improved renal function parameters obtained in this study are attributable to the natural recovery process of AKI or the efficiency of fenoldopam. To address this issue, multicentre randomized controlled trials with a large sample size are still needed.

\section{Compliance with ethical standards}

Conflict of interest All authors have no financial support and potential conflicts of interest for this work.

\section{References}

1. Biancofiore G, Bindi ML, Miccoli M, Cerutti E, Lavezzo B, Pucci L, Bisà M, Esposito M, Meacci L, Mozzo R, Stratta C, Penno G, Baggiani A, Filipponi F. Intravenous fenoldopam for early acute kidney injury after liver transplantation. J Anesth. 2015;29:426-32.

2. Coca SG, King JT Jr, Rosenthal RA, Perkal MF, Parikh CR. The duration of postoperative acute kidney injury is an additional parameter predicting long-term survival in diabetic veterans. Kidney Int. 2010;78:926-33. 